\title{
Examination of Drone Micro-Doppler and JEM/HERM Signatures
}

\author{
John Markow and Alessio Balleri \\ Centre for Electronic Warfare, Information and Cyber, Cranfield University \\ Defence Academy of the UK, Shrivenham, SN6 8LA \\ jmm0006@uah.edu a.balleri@cranfield.ac.uk
}

\begin{abstract}
Radars monitoring small targets often must increase their integration times to achieve sufficient signal-to-noise ratio (SNR) for maintaining a viable track. These longer integation times can prevent micro-Doppler signature extraction and instead result in Doppler signatures consisting of spectral lines to the radar's higher-level processing. Whether the radar operates in the micro-Doppler or spectral line regime depends on both radar parameters (e.g. waveforms, wavelengths and integration times) as well as target parameters (e.g. rotor length, rotational frequency, target reflectivity and geometry). Additionally, understanding the transition region between these regimes can further aid target recognition algorithms. This paper uses modelling, simulations and experimental data to refine the understanding of how a particular radar will observe a target Doppler signature in either of these regimes, highlighting the transition region between the two.
\end{abstract}

Keywords-micro-Doppler, target signature, jet engine modulation (JEM), helicopter rotational motion (HERM), spectrogram, drone

\section{INTRODUCTION AND LITERATURE REVIEW}

As modern radars are designed to perform an increasing number of tasks, they encounter an increasing number of target types. Among these are targets that produce either JEM/HERM and/or micro-Doppler signatures. Therefore, radar designers developing Doppler-based target recognition algorithms must have nuanced understandings of target signatures in both regimes to select the most appropriate algorithms. This paper reviews Doppler signature theory for rotating scatterers and uses experiments and modelling to investigate and illustrate a variety of these time-frequency signatures, using short-time Fourier transform (STFT) processing, paying particular attention to the transition region between the micro-Doppler and JEM/HERM regimes.

Researchers have been examining Doppler signatures arising from jet engine modulation (JEM) and helicopter rotation motion (HERM) for decades [1-3]. A widely-cited approach presented in [1] characterises both the time-domain and frequency-domain response of a radar viewing a rotating propeller, highlighting the relevant variables and providing rules of thumb for predicting frequency spacing and overall expanse of spectral lines. Another widely-cited source provides a foundational approach to micro-Doppler analysis and signature prediction by developing the mathematical theory and models and comparing them to collected data [4]. Expanding these concepts further, [5-9] apply them to different target types and present different processing methods. As for micro-Dopplerbased target classification, [10-18] look at a number of different target types and present a wide variety of classification strategies and algorithms.
While the body of work on Doppler signatures of rotating or oscillating objects, whether they be propellers, rotors, bird wings or platform oscillations, is broad and diverse, the sources cited above pay little attention to the transition zone between micro-Doppler and JEM/HERM regimes and the factors affecting this zone. To that end, this research looks at modelling a single target Doppler signature on either side of the transition region to see how key target features affect the transition from one regime to the other. Understanding the features that manifest in the micro-Doppler regime, conditions precluding micro-Doppler processing and/or how to coordinate target recognition algorithms on either side of the transition region can help radar designers select appropriate algorithms in a more nuanced manner. Additionally, this research captures the impacts of radar design decisions, highlighting the need to appropriately apply different classification algorithms for either regime. Throughout, experimental data is presented and simulated data is used as needed.

\section{BACKGROUND THEORY}

A rotating object creates a time-varying Doppler shift to a radar that is a function of many variables. Drawing from [1] and [4], a single scatterer rotating around a centre of rotation can be illustrated as shown in Figure 1.

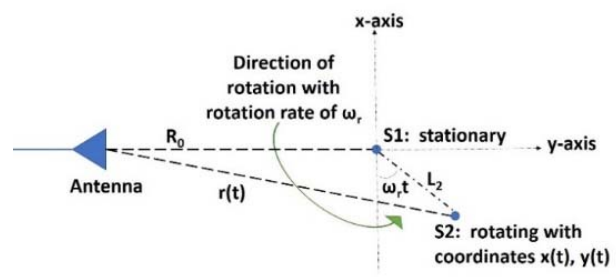

Figure 1: Rotating Scatterer Model

In Figure $1, \mathrm{R}_{0}$ is the range from the radar antenna to a stationary scatterer, $\mathrm{S} 1 ; \mathrm{r}(\mathrm{t})$ is the time-varying range from the antenna to a scatterer, $\mathrm{S} 2$, which is rotating around $\mathrm{S} 1 ; \omega_{r}$ is the rotational rate of $\mathrm{S} 2$; and $\omega_{r} t$ is the angle at time $t$ that the radius of rotation, $L_{2}$, forms with the $\mathrm{x}$-axis $\left(\mathrm{L}_{2}\right.$ is the fixed distance of $\mathrm{S} 2$ from the centre of rotation). This model ignores any initial scatterer angle and assumes the radar and rotating scatterer are in the same plane. The coordinates of $\mathrm{S} 2$ relative to the origin are $x(t)=L_{2} \cos \omega_{r} t$ and $y(t)=L_{2} \sin \omega_{r} t$. Using the Pythagorean theorem and assuming $L_{2} \ll R_{0}$, the range from the antenna to $\mathrm{S} 2$ is $r(t)=R_{0}+L_{2} \sin \left(\omega_{r} t\right)$ [4]. If the radar transmits an unmodulated signal, neglecting the amplitude term, the phase modulated component of the reflected signal from the rotating scatterer is given by

$$
s_{r}(t)=e^{j\left(2 \pi f_{c} t-\Phi(t)\right)}
$$

where $f_{c}$ is the carrier frequency ( $\lambda$ is the wavelength) and

$$
\Phi(t)=\frac{4 \pi}{\lambda}\left(R_{0}+L_{2} \sin \left(\omega_{r} t\right)\right) .
$$


If the scatterers are moving along the y-axis, a velocity term, $v$, contributes a time-varying phase shift (note a positive velocity corresponds to the reflectors moving away from the radar). Additionally, if the scatterers are not coplanar with the antenna (i.e., if S1 and/or S2 are displaced along a z-axis), the angle $\theta$, capturing the angle between the radar's line-of-sight (LOS) to the target and the rotating scatterer's plane of rotation, must be included in (2), and the final mathematical expression becomes

$$
\Phi(t)=\frac{4 \pi}{\lambda}\left(R_{0}+v t+\cos (\theta) L_{2} \sin \left(\omega_{r} t\right)\right) .
$$

As a result, the instantaneous frequency, $f_{i}(t)$, of the received signal is

$$
f_{i}(t)=-\frac{2}{\lambda}\left(v+L_{2} \omega_{r} \cos (\theta) \cos \left(\omega_{r} t\right)\right) .
$$

The signal model in (1) can be expanded to account for $N$ finite-length propeller blades and for the scattering properties of a distributed reflection (i.e., a helicopter rotor or jet-engine compressor blade). The time-domain return signal for this target type is given as $[1,3]$

$$
\begin{aligned}
& v_{r}(t)=\sum_{n=0}^{N-1} e^{j\left(\omega_{c} t-\frac{4 \pi}{\lambda}\left[\begin{array}{c}
A_{r}\left(L_{2}-L_{1}\right) \\
R_{0}+v t+\frac{L_{2}+L_{1}}{2} \cos (\theta) \\
\sin \left(\omega_{r} t+\frac{2 \pi n}{N}\right)
\end{array}\right]\right)} \\
& \operatorname{sinc}\left(\begin{array}{c}
\frac{4 \pi}{\lambda} \frac{L_{2}-L_{1}}{2} \cos (\theta) \\
\sin \left(\omega_{r} t+\frac{2 \pi n}{N}\right)
\end{array}\right)
\end{aligned}
$$

In (5), $A_{r}$ is a scale factor, $L_{1}$ is the distance from the centre of rotation to the blade root, $\omega_{c}=2 \pi f_{c}$ is the carrier frequency in $\mathrm{rad} / \mathrm{s}$. This equation captures the critical variables shaping the Doppler signature of a rotating scatterer(s).

Isolating a single rotating point scatterer provides insight into the transition zone between a micro-Doppler signature and JEM/HERM lines. Simply put, if the integration time of the radar's Doppler processor is significantly less than the rotational period of a single point scatterer, then a microDoppler signature can be extracted; if it is significantly longer, spectral lines will occur. Figure 2 illustrates this point by sweeping the integration time of $e^{-j \sin \left(\omega_{r} t\right)}$ (which corresponds to the term $e^{-j \Phi(t)}$ for a fixed value of $\omega_{r}$ when $\frac{4 \pi}{\lambda} L_{2}$ is specifically set to unity), in order to isolate the sinusoidally varying phase shift, from $1 / 100^{\text {th }}$ of a rotation to five times the rotational period. The highest expected Doppler shifts are negative and positive $f_{r}=w_{r} /(2 \pi)$.

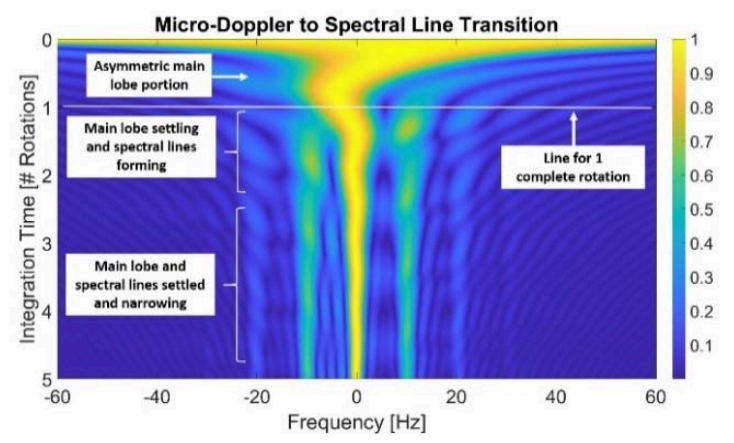

Figure 2: Rotating Scatterer Model
As the image moves from the minimum integration time to about half of a rotation, a ridge emerges asymmetrically. Its initial formation depends on the initial phase of the rotating scatterer. In this regime, no periodic information is available to the processor, hence no spectral lines form. After one rotation, the central spectral line settles at $0 \mathrm{~Hz}$ and around the second rotation, additional spectral lines start to form. In this simulation, the single scatterer had a rotational frequency of $10 \mathrm{~Hz}$, hence when the spectral lines do form, they occur at positive and negative multiples of $10 \mathrm{~Hz}$. As expected, for the lowest integration times, the frequency resolution is much higher than the Doppler returns from the scatterer and no Doppler information can be extracted. Beyond this case, the largest observable Doppler shift occurs when the scatterer reaches the point of maximum radial velocity within the first rotation. At this point, the Doppler is slightly less than the rotational frequency of $f_{r}=10 \mathrm{~Hz}$ since the frequency shown in the figure is the average of the instantaneous frequencies that occur during the integration window and because the expected Doppler shifts are comparable with the frequency resolution. Since the relationship between integration time and maximum observed frequency is highly phase dependent, two coherent dwells can produce two different spectra on the same target if the integration time is fractions of the rotational period. Once the integration time is much longer than the rotational period, the integration averages all the instantaneous frequencies together, producing the main lobe at $0 \mathrm{~Hz}$. Finally, the spectral lines are seen to narrow as the integration time increases, as expected.

\section{EXPERIMENTAL AND SimUlATED DATA}

To verify the theory above, experimental data was collected in order to create both a micro-Doppler signature as well as a spectral line plot. Simulations were built based on the theory and compared to the experimental data. Then, for cases that could not be tested in the laboratory, simulations were used to predict further Doppler signatures.

\section{A. Experiments}

Experiments and simulations were conducted to better understand this theory. In the first example, a two-bladed helicopter drone was selected as it was likely to produce a micro-Doppler signature. It was mounted to a stand, placed in the far field of a pulsed radar using 5-MHz chirp waveforms and reflection enhancers were placed on the rotors. This experiment was conducted at two different frequencies and an image of the setup is shown in Figure 3.

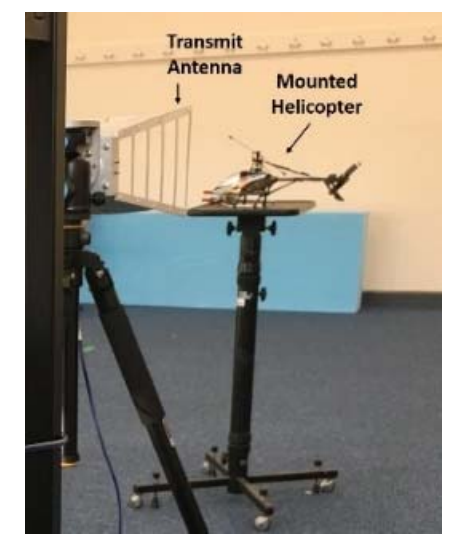

Figure 3: Helicopter Drone Experiment Setup Table I.

The critical parameters of the experiment are given in 
TABLE I. HELICOPTER DRONE EXPERIMENT SETUP

\begin{tabular}{|c|c|c|c|}
\hline Parameter & Symbol & Values & Units \\
\hline Rotor maximum length & $L_{2}$ & 18 & $\mathrm{~cm}$ \\
\hline Rotor minimum length & $L_{1}$ & 0.5 & $\mathrm{~cm}$ \\
\hline Rotational frequency & $f_{r}$ & Approx. 15 & $\mathrm{~Hz}$ \\
\hline Wavelength & $\lambda$ & 6 and 10 & $\mathrm{~cm}$ \\
\hline Carrier Frequency & $f_{c}$ & 5 and 3 & $\mathrm{GHz}$ \\
\hline Number of rotors & $N$ & 2 & $+/ \mathrm{N} / \mathrm{A}$ \\
\hline Dwell time & $t_{D}$ & 500 and 250 & $\mathrm{msec}$ \\
\hline
\end{tabular}

Figure 4 shows the experimental results obtained at $6 \mathrm{GHz}$. As theory predicts $\left(f_{\text {Doppler }}=2 \omega_{r} L_{2} / \lambda\right)$, the spectral extent of the rotor blades spans approximately $+/-565 \mathrm{~Hz}$. One blade was more reflective than the other, hence the asymmetric spectrogram. In the approximately $500 \mathrm{msec}$ dwell time, the rotor completed approximately 6.5 rotations, consistent with a $15 \mathrm{~Hz}$ rotation rate. Also, the STFTs to create the spectrograms were 100 points long, which at a PRF of $4 \mathrm{kHz}$ requires $25 \mathrm{msec}$ of time. Given the $66.6 \mathrm{msec}$ rotational period of the blades, they only accomplish $37.5 \%$ of a rotation during one STFT window.

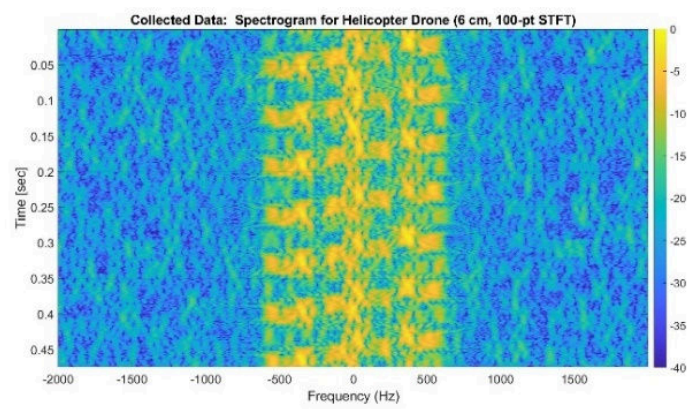

Figure 4: Experimental Helicopter Drone Spectrogram

Figure 5 shows the results obtained with a MATLAB simulation that recreated the laboratory data in order to increase the credibility of the modelling approach for later use in cases where experimental data was unavailable. Asymmetric rotor reflection coefficients were included to match the experimental data (one rotor was modelled with $3 / 4$ the reflectivity of the other). The spectral extent and number of rotations match the experimental data, although underlying body vibrations were not modelled, hence the much 'cleaner' spectrogram.

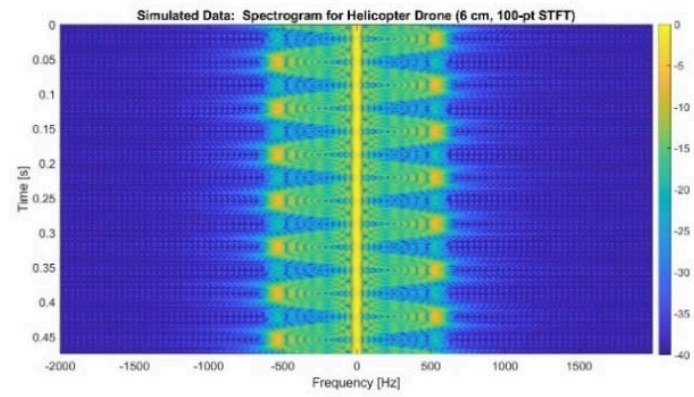

Figure 5: Simulated Helicopter Drone Spectrogram

The experiment was repeated at $3 \mathrm{GHz}(10 \mathrm{~cm}$ wavelength) (for this experiment, the dwell was reduced by half compared to the $6 \mathrm{GHz}$ case). Again, consistent with theory, the spectral extent of the rotor blades spans $+/-339 \mathrm{~Hz}$.

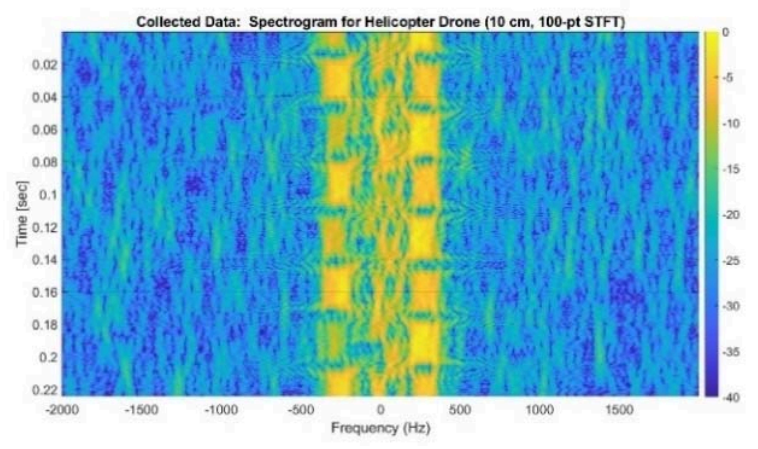

Figure 6: Experimental Helicopter Drone Spectrogram

In the simulated data shown in Figure 7, the spectral spreading and sinusoidal pattern match closely to Figure 6, although the simulation did not account for the asymmetric reflectivity of the rotor blades.

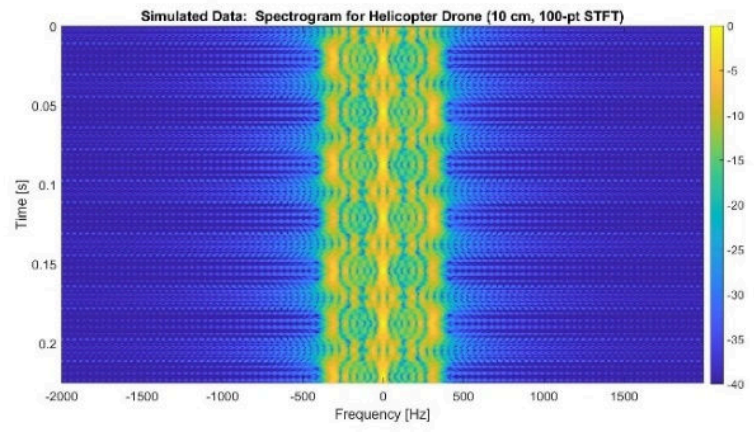

Figure 7: Simulated Helicopter Drone Spectrogram

Returning to the experimental data, had the integration time been slightly longer, 200 points, or $75 \%$ of a rotation, the micro-Doppler signature from Figure 6 starts transitioning into spectral lines. Results for the $3 \mathrm{GHz}$ case are shown in Figure 8.

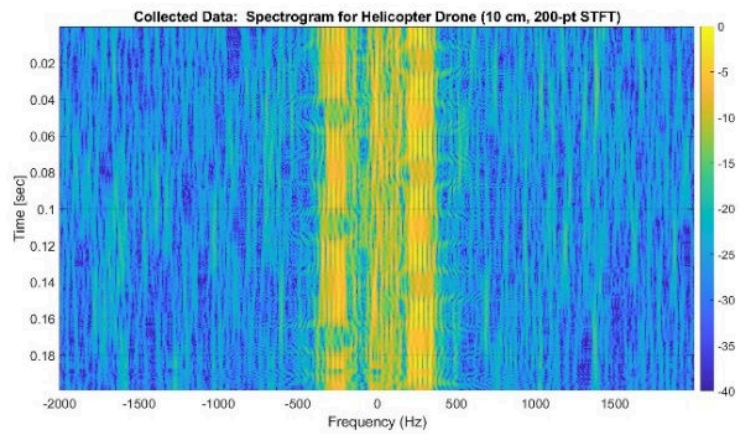

Figure 8: Experimental Helicopter Drone Spectral Lines

Next, a similar experiment was conducted with a quadcopter. The target was at approximately 4-5 meters from the radar, the rotational frequency of the blades varied about $80 \mathrm{~Hz}$ and the blade lengths were $11.5 \mathrm{~cm}$. In order to achieve sufficient integration gain to form a useable spectrogram, the STFT window was 800 points (well more than the 100 points used in the helicopter case), which at a PRI of $250 \mu \mathrm{sec}$ corresponds to a dwell time of $200 \mathrm{msec}$. For a rotational frequency of approximately $80 \mathrm{~Hz}$, the rotors completed around 16 rotations during the STFT window, preventing the generation of sinusoidal micro-Doppler signatures. 
Therefore, a simple range-Doppler map (RDM) was used to present the data.

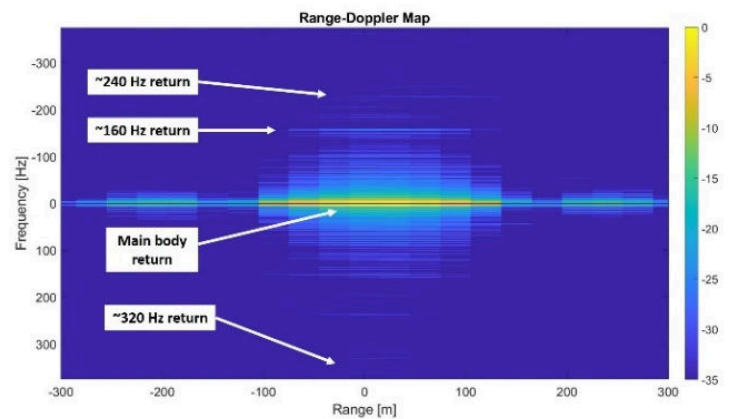

Figure 9: Experimentally-Collected Quadcopter RDM

In Figure 9, the experimental data shows spectral lines at multiples of the rotational frequency of $80 \mathrm{~Hz}$, though not all spectral lines emerged from the noise. The spectral lines occur at multiples of $80 \mathrm{~Hz}$ vice $160 \mathrm{~Hz}$ because of the asymmetric reflectivity of the rotors presenting different signal returns at the rotational velocity. Further, the spectral lines are broadened and slightly shifted higher or lower in some cases because the variances of the rotational frequencies ranged from the single digits to the low twenties $\mathrm{Hz}^{2}$. For example, the $320 \mathrm{~Hz}$ line is faintly spread on approximately 310 and $330 \mathrm{~Hz}$. Additionally, electromagnetic wave reflection about the quadcopter led to additional signature variations.

In the simulation, shown in Figure 10, the rotational frequencies were generated with a mean of $80 \mathrm{~Hz}$ and a standard deviation of $+/-5 \mathrm{~Hz}$.

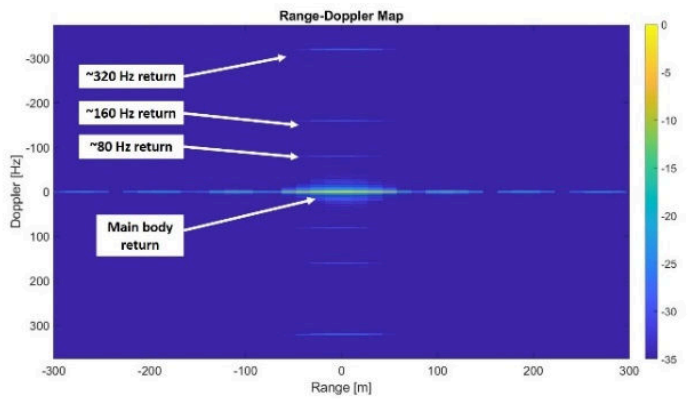

Figure 10: Simulated Quadcopter RDM

These experiments confirmed the theory presented above that relates the integration time to rotational frequency to predict the category of signature (micro-Doppler or spectral line) that a given radar and target will produce. It also highlighted the impacts of real-world phenomena such as platform motion, rotational frequency variation and range attenuation.

\section{B. Further Simulations}

Since many scenarios cannot be recreated in the laboratory, simulation must be used to predict their Doppler signatures. This research built a MATLAB simulation of (5) and swept various target and radar parameters to better understand the interdependence of these variables in shaping the Doppler signatures. After the JEM/HERM signatures were explored, the transition region for a quadcopter was examined.

As seen in [1], it is possible to estimate the number and locations of spectral lines for a JEM/HERM-producing target, in other words, a target whose rotating scatterer(s) completes multiple rotations within one integration period. Therefore, parameterising key variables of (5) allows for easy prediction of Doppler signatures. For example, Figure 11 shows parametrisation of the rotor length to wavelength ratio, as in $\frac{4 \pi}{\lambda} L_{2} \cdot{ }^{1}$ The output illustrates how many spectral lines should be expected for a given ratio. The second sinc term in (5) acts as an envelope bounding the onset of spectral lines at lower frequencies and their cessation at higher frequencies [3].

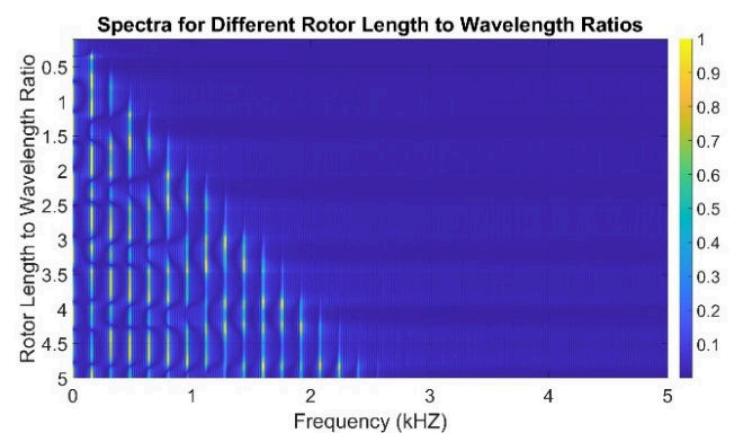

Figure 11: Signatures Variation with Rotor Length to Wavelength Ratio

The rotor length to wavelength ratio attenuates or accentuates the phase shifts that arise from rotating scatterers as well as role of the sinc term. The latter in particular captures the extent to which the propeller can be treated as a line antenna of finite length. Consistent with electromagnetics theory, the gain of an antenna is inversely related to the square of the wavelength, so if the wavelength increases, the antenna gain, or, in this case, the energy reflected from the propeller in the direction of the receiver, decreases geometrically [3].

Figure 12 shows Doppler spectral variation of the same radar/target with rotational frequency. As the rotational frequency increases, the spectral lines move linearly away from each other as the maximum imparted Doppler shift increases with greater rotational velocity. A longer radius of rotation would also shift these spectral lines higher in frequency.

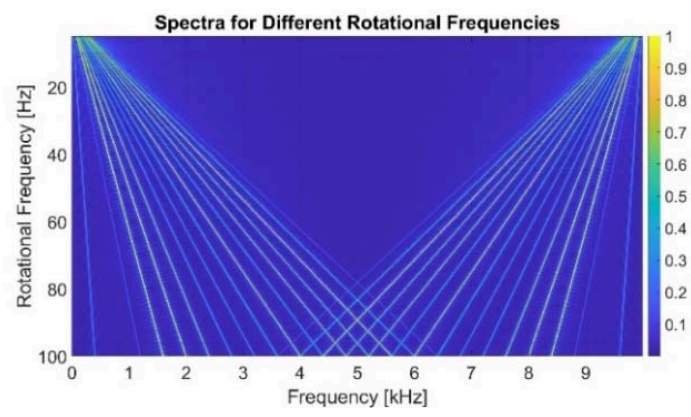

Figure 12: Signature Variation with Rotational Frequency

After examining some basic parameterisation, a more involved simulation was conducted that combined multiple
${ }^{1}$ Figures 11 and 12 were created using the same values (except the parameterised variable) as [1]: $10 \mathrm{kHz}$ sampling frequency (PRF), $50 \mathrm{msec}$ dwell, a $40 \mathrm{~Hz}$ rotational frequency, $L_{2}$ set to $1 \mathrm{~m}$ and $L_{1}$ set to 0 , four point scatterers and $\theta$ set to zero degrees. 
changing variables. In this scenario, a quadcopter was modelled flying for 100 seconds. A pulsed radar was modelled and the Doppler signature over the trajectory was determined. Table II provides critical parameters.

TABLE II. QUADCOPTER SIMULATION

\begin{tabular}{|c|c|c|c|}
\hline Parameter & Symbol & Values & Units \\
\hline Rotor maximum length & $L_{2}$ & 7 & $\mathrm{~cm}$ \\
\hline Rotor minimum length & $L_{1}$ & 0.5 & $\mathrm{~cm}$ \\
\hline Body velocity & $v$ & Approx. -5 to 15 & $\mathrm{~m} / \mathrm{s}$ \\
\hline Rotational frequency & $f_{r}$ & $16.5-35$ & $\mathrm{~Hz}$ \\
\hline Wavelength & $\lambda$ & 25 & $\mathrm{~cm}$ \\
\hline Carrier Frequency & $f$ & 1.2 & $\mathrm{GHz}$ \\
\hline Number of rotors & $N$ & 4 & $\mathrm{~N} / \mathrm{A}$ \\
\hline Dwell time & $t$ & 250 & $\mathrm{msec}$ \\
\hline LOS Angle & $\theta$ & $0-90$ & $\mathrm{deg}$. \\
\hline FFT Length & N/A & 2048 & $\mathrm{pulses}$ \\
\hline Pulse Repetition Frequency & PRF & 8 & $\mathrm{kHz}$ \\
\hline
\end{tabular}

Over the course of the flight, the quadcopter's bulk velocity and the rotors' rotational frequency varied quasisinusoidally while the LOS angle increased linearly from zero to 90 degrees.

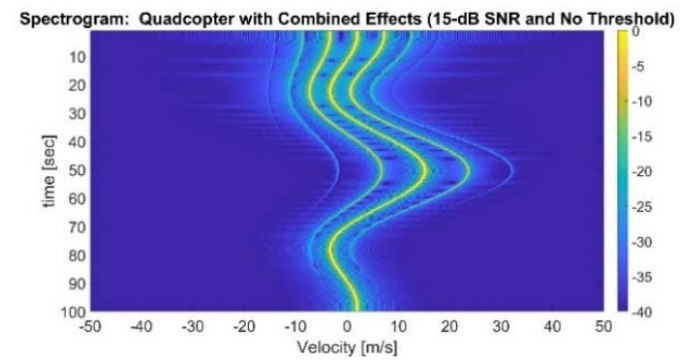

Figure 13: Signature Variation due to Multiple Variable Changes

Initially five spectral lines are present and as the rotational frequency increases, they drift apart (again, they have their own rotational velocity variation). Additionally, as the LOS angle approaches 90 degrees, the spectral lines collapse down into the central scatterer's line (note, blade chopping was not modelled in this scenario which would most likely have allowed some spectral lines to persist).

Next, using the same simulation scenario, an SNR of 15 $\mathrm{dB}$ and a threshold of $10 \mathrm{~dB}$ were applied giving yet a different signature, relevant for automatic target recognition cases.

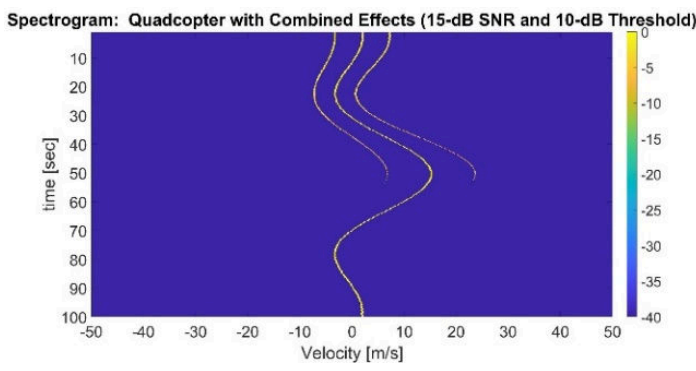

Figure 14: Signature Variation due to Multiple Variable Changes

The noise and threshold produce noticeably different target signatures at different points in the trajectory. The thresholding erases the FFT sidelobes present in Figure 13, so the spectral lines appear clearer, which could help with target recognition algorithms. Additionally, as the LOS angle approaches 90 degrees, the spectral lines collapse into the centre scatterer, slightly increasing its SNR.

While parameterising variables of (5) helps predict where the spectral lines will fall once they form, it is useful to try to predict how the micro-Doppler and transition regions will appear. To this end, a simulation of the same quadcopter from Table II (with $f_{r}$ set to $20 \mathrm{~Hz}$ ) viewed for varying integration times is presented in Figure 15. Given the use of symmetric two-bladed rotors and constant $f_{r}$, the spectral lines fall at twice $f_{r}$.

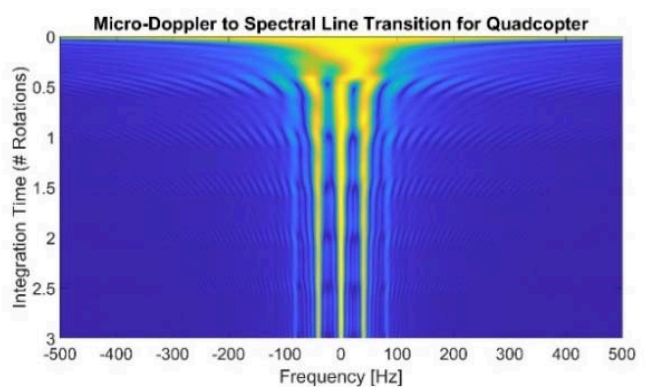

Figure 15: Rotating Scatterer Model (1.2 GHz)

Figure 15 is similar to Figure 2 but highlights the specific case of the four-bladed quadcopter (also, only three rotations are shown vice five). Like Figure 2, the blades create broad, asymmetric ridges in the micro-Doppler regime however here they abruptly splinter into additional ridges around half of a rotation (these rotors are simulated as two point-scatterers diametrically opposite compared to the single point scatterer in Figure 2). The nature of this transition from a single ridge to a multi-ridge is phase-dependent - the specific time and shape of it varies with initial phase. Ultimately, during the micro-Doppler regime, the ridges settle into three independent ridges at the first multiple of the rotational frequency. This figure illustrates that had micro-Doppler processing of the target been desirable and possible, integration times up to about half of a rotation would have provided sufficient timevarying Doppler to identify rotational motion. Past the microDoppler regime, the additional blades facilitate quicker spectral line narrowing compared to the single scatterer case shown in Figure 2.

Two final figures reiterate that the transition regime is also dependent on other variables. Here, a different rotor length-towavelength ratio is chosen. As shown in Figure 11, as this ratio grows (either longer rotors or shorter wavelengths), the number of spectral lines increases. In the plots below, the rotor length from Table II is used with a $2 \mathrm{GHz}$ (15-cm wavelength) and a $6 \mathrm{GHz}$ (5-cm wavelength) radar, corresponding to ratios of 0.466 and 1.4 , respectively.

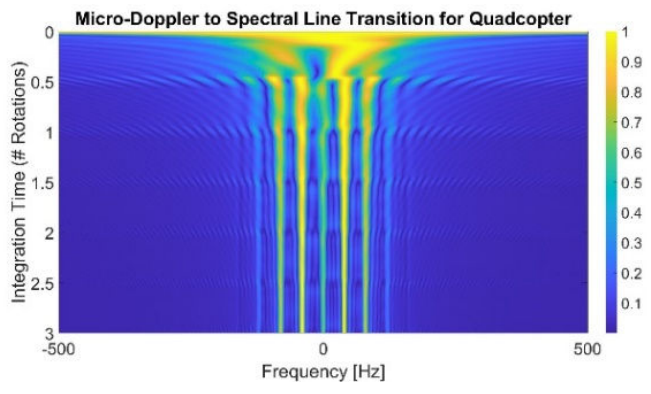

Figure 16: Rotating Scatterer Model (2 GHz) 
While the rotor length-to-wavelength ratio is less than approximately 0.5 , one main ridge dominates the microDoppler regime, prior to the formation of spectral lines. Once the ratio exceeds one half, two major ridges along with smaller ridges dominate the regime. Finally, whereas in Figure 16, the micro-Doppler ridge peaks in frequency around $90 \mathrm{~Hz}$ (slightly less than the maximum expected Doppler of $117 \mathrm{~Hz}$ ), at $6 \mathrm{GHz}$, two very large ridges dominate at approximately $+/-$ $310 \mathrm{~Hz}$, also slightly less than the maximum anticipated Doppler shift.

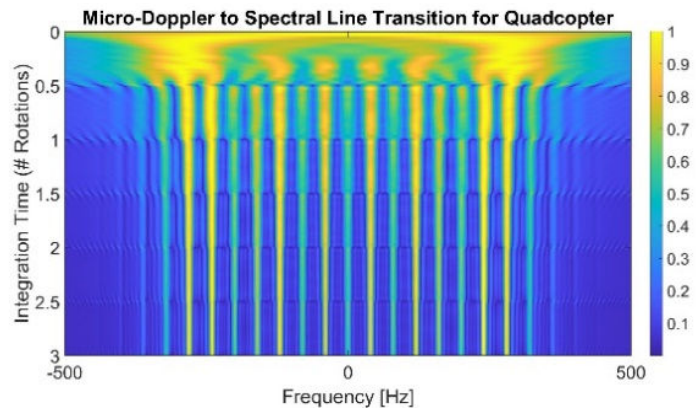

Figure 17: Rotating Scatterer Model (6 GHz)

Figures 16 and 17 highlight not only the notable variation in spectral line formation with wavelength, but also the different micro-Doppler signatures. Additionally, the initial phases of the rotors significantly shape the spectrum prior to $1 / 2$ of a rotation. If automatic target recognition algorithms are being developed to operate in the micro-Doppler regimes for quadcopters like the ones discussed in this research, reference libraries and training data must take these dynamic signatures into account.

\section{CONCLUSIONS}

This paper presented Doppler spectral analysis applied to rotating scatterers such as helicopter and quadcopter drones. The goal was to better understand how the variables of a radar and a target shape the transition between the micro-Doppler and JEM/HERM regimes when performing Doppler processing. Experimental data was collected to bolster theory on the topic and simulations were used where experiments were not possible. The findings of this research illustrate the significance of the STFT length as well as the highly dynamic signature fluctuations in the micro-Doppler regime. Welltailored target recognition algorithms would take these factors into account by adjusting the STFT length, incorporating target signatures from variable-length STFTs in target databases and comparing data from both sides of the transition region all to enhance identification abilities.

\section{Acknowledgements}

The authors wish to thank Professor Chris Baker and Dr. Mohammed Jahangir for providing comments and points of discussion during this research. John Markow is a graduate of the Guided Weapons MSc course at Cranfield University, Defence Academy of the United Kingdom, Shrivenham, UK.

\section{REFERENCES}

[1] J. Martin and B. Mulgrew, "Analysis of the theoretical return signal from aircraft propeller blades," IEEE International Radar Conference, pp. 569-572, 1990.

[2] M. Bell and R. Grubbs, "JEM modelling and measurement for radar target identification," IEEE Transactions on Aerospace and Electronic Systems, vol. 29, No. 1, pp. 73-87, 1993.

[3] A. French, "Target recognition techniques for multi-function phase array radar," University College London, PhD Thesis (unpublished), 2010, Available at http://discovery.ucl.ac.uk/19675/1/19675.pdf (Accessed 24 May 2019).

[4] V. Chen, "The micro-Doppler effect in radar," $2^{\text {nd }}$ edn, Boston, Artech House 2019.

[5] S. Gorbuz, C. Clemente, A. Balleri and J. Soraghan, "Micro-Dopplerbased in-home aided and unaided walking recognition with multiple radar and sonar systems," IET Radar, Sonar and Navigation, vol 11, Issue 1, 2017, pp. 107-115.

[6] A. Balleri, K. Chetty and K. Woodbridge, "Classification of personnel targets by acoustic micro-Doppler signatures," IET Radar, Sonar and Navigation, vol. 5, iss. 9, 2011, pp. 943-951.

[7] P. Addabbo, C. Clemente and S. Ullo, "Fourier-independent component analysis of radar micro-Doppler features," IEEE International Worskshop on Metrology for AeroSpace, 2017, pp. 4549, Available

https://strathprints.strath.ac.uk/64084/1/Addabbo_etal_MetroAeroSpa ce 2017 Fourier independent component analysis of radar micro Doppler_features.pdf (Accessē 24 May 2019).

[8] A. Brewster and A. Balleri, "Extraction and analysis of micro-Doppler signatures by the emperical mode decomposition," IEEE Radar Conference, Arlington, VA, 2015, pp. 947-951.

[9] T. Thayaparan, et. al., Analysis of radar micro-Doppler signatures from experimental helicopter and human data," IEEE Proceedings on Radar, Sonar and Navigation, vol 1, no. 4, 2007, Available at http://www.tfsa.ac.me/pap/15_3.pdf (Accessed 24 May 2019).

[10] D. Blackness and H. Griffiths (eds), "Radar automatic target recognition (ATR) and non-cooperative target recognition (NCTR)," London, Institute of Engineering and Technology, 2013.

[11] V. Nebabin, "Methods and techniques of radar recognition," D. Barton Ed., Boston: Artech House, 1995.

[12] S. Park, "Automatic target recognition using jet engine modulation and time-frequency transform," Progress in Electromagnatics Research, vol. 39, 2014, pp. 151-159, Available at: www.jpier.org/PIERM/pierm39/16.14100701.pdf (Accessed 25 June 2019).

[13] S. Nguyen, S. Kodituwakku, R. Melino and H. Tran, "Signal separation of helicopter radar returns using wavelet-based sparse signal optimisation, Edinburgh, South Australia, Defence Science and Technology Group (unpublished), 2016. Available at https://www.dst.defence.gov.au/publication/signal-separationhelicopter-radar-returns-using-wavelet-based-sparse-signal (Accessed 24 May 2019).

[14] S. Rahman and D. Robertson, "Radar micro-Doppler signatures of drones and birds at K-band and W-band," Nature: Scientific Reports, vol. 8, Article Number 17396, 2018, available at: https://www.nature.com/articles/s41598-018-35880-9 (Accessed 25 June 2019).

[15] B. Torvik, "Investigation of non-cooperative target recognition of small and slow moving air targets in modern air defense surveillance radar," London: University College London (unpublished); 2016, available at discovery.ucl.ac.uk/1532017/1/Torvik_2016-1213_PhdThesisTorvik.pdf (Accessed 24 May 2019).

[16] M. Jahangir, C. Baker and G. Oswald, "Doppler characteristics of micro-drones with L-band multibeam staring radar," IEEE Radar Conference, 2017, pp. 1052-1057.

[17] V. Chen, "Analysis of radar micro-Doppler signature with timefrequency transform," Proceedings of the $10^{\text {th }}$ IEEE Workshop on Statistical Signal and Array Processing; Pocono Manor, PA, USA; 2000, pp. 463-466.

[18] J. Patel, F. Fioranelli and D. Anderson, "Review of radar classification and RCS characterization techniques for small UAVs or drones," IET Radar, Sonar and Navigation, vol. 12; issue 9, pp. 911-919. 